\title{
In situ studies of cellular architecture by Electron Cryo-Tomography with Volta Phase Plate
}

Yoshiyuki Fukuda ${ }^{1}$, Shoh Asano ${ }^{1}$, Ulrike Laugks ${ }^{1}$, Florian Beck ${ }^{1}$, Antje Aufderheide ${ }^{1}$, Friedrich Förster ${ }^{1}$, Vladan Lučić ${ }^{1}$, Wolfgang Baumeister ${ }^{1}$, Radostin Danev ${ }^{1}$.

1. Department of Molecular Structural Biology, Max Planck Institute of Biochemistry, Martinsried, Germany.

Studies of molecular sociology of cells and in situ studies of macro molecular assemblies are critical for our understanding of cellular function. Electron cryo-tomography (ECT) of vitrified, frozen-hydrated cells provides a means of studying the three dimensional structure of pleomorphic objects, such as organelles or cells preserved in their natural, cellular environment, with a resolution of 1 to $3 \mathrm{~nm}$ range [1]. However, low signal-to-noise ratio in image is a drawback of ECT.

In order to improve the image contrast of frozen-hydrated specimens, several kinds of phase plates have been developed [2]. Among them, Zernike type phase plate has been used for studies of frozen-hydrated biological specimens in previous studies [3,4]. Although Zernike type phase plate is possible to enhance image contrast but it also generates fringes around structures which make interpretation of structure difficult [5]. Recently, new type of phase plate for TEM named Volta phase plate (VPP) was developed [6]. In this study, we applied VPP to ECT of frozen-hydrated cells such as magnetotactic bacteria and primary cultured neuronal cells for visualizing details of molecular architecture in situ.

In order to investigate the performance of the VPP for thick specimens we observed plunge-frozen magnetotactic bacteria. Four tomographic tilt-series were acquired with different combinations of an energy filter and a VPP. Energy filtered images show stronger contrast for large objects, such as the poly- $\beta$-hydroxybutyrate (PHB) granules, and less background noise in the thicker parts of the sample than the non-filtered images. In comparison, the VPP improves the contrast of all visible features.

As a next step, we compared the quality of reconstructed tomograms. Similarly to the projection images, these slice images show that the energy filter improves the contrast of large objects, such as the PHBs. The contrast of inner and outer membranes was also slightly improved by the energy filtering. Surprisingly, the effect of zero-loss filtering on the overall contrast in reconstructed tomograms is diminished compared to that in the tilt-series images. Comparing the CTEM slices with the VPP ones the visibility and contrast of many intracellular structures and macromolecules was significantly improved by the VPP. For example, the chemoreceptor arrays are easier to detect in both the unfiltered and filtered VPP tomograms than in the energy filtered CTEM tomogram. Therefore, the VPP produces a significant increase in contrast, in addition to that provided by energy filtering.

In order to quantitatively evaluate the contrast of the inner and outer membranes for the different acquisition conditions we calculated intensity profiles across the membranes. To make the comparison as unbiased as practically possible, considering the tomograms were acquired at different specimen areas, the profiles were calculated at a position which showed approximately the same cell. The energy filter provided $\sim 16 \%$ contrast increase without a VPP and $\sim 22 \%$ with a VPP. On the other hand the VPP provided $\sim 61 \%$ contrast improvement without and $\sim 68 \%$ with an energy filter. 
Due to the improvement of visibility provided by VPP, 26S proteasome particles could be visualized in ECT of primary cultured neuronal cells. For the in situ studies of macro molecular structure, we attempted template matching using a 3D model of single-capped $26 \mathrm{~S}$ proteasome and sub-tomogram averaging of matched particles. Two of major conformational states "ground state" and "substrateprocessing state" are obtained by classification.

Thus, VPP provides ECT of frozen-hydrated specimens a significant improvement of contrast for visualization of cellular architecture and macro molecular structure in situ. We expect that VPP will become a standard element of the electron cryo-tomography workflow.

\section{References:}

[1] V Lučić, A Rigort and W Baumeister, J. Cell Biol., 202 (2013), p. 407.

[2] R M Glaeser, Rev. Sci. Instrum., 84 (2013), 111101.

[3] R Danev, R M Glaeser, K Nagayama, Ultramicroscopy, 109 (2009), p. 312.

[4] W Dai, et al, Nature, 502 (2013), p. 707.

[5] R Danev and K Nagayama, Ultramicroscopy, 111 (2011), p. 1305.

[6] R Danev et al, Proc. Natl. Acad. Sci. USA., 111 (2014), p. 15635.
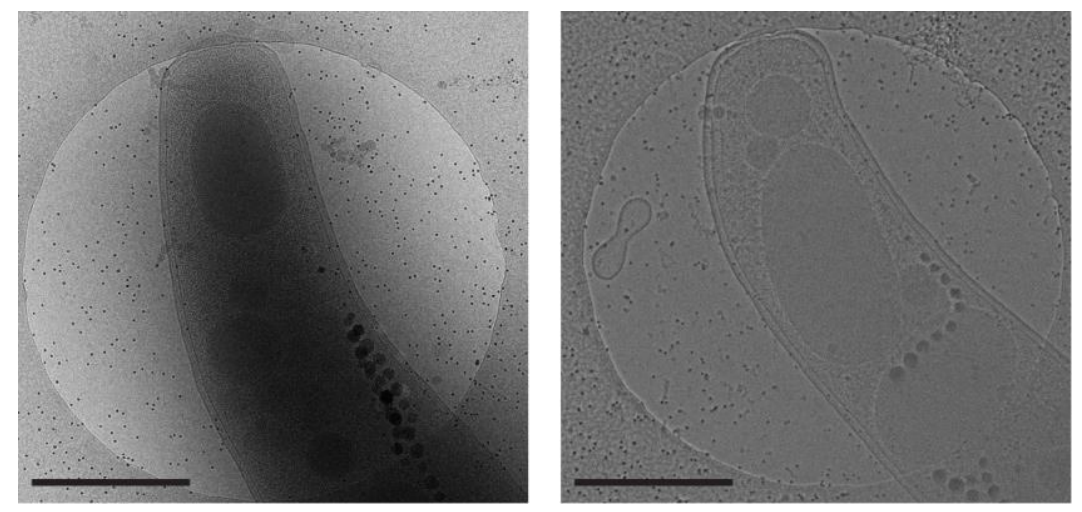

Figure 1. Comparison of projection images of plunge-frozen Magnetotactic bacteria between CTEM with energy filter and VPP without energy filter. Scale bars: $500 \mathrm{~nm}$.
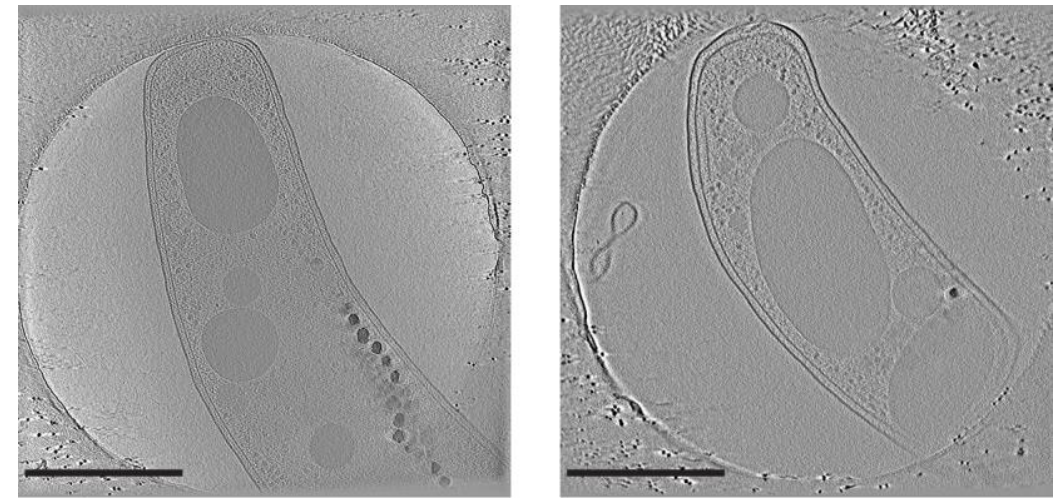

Figure 2. Comparison of tomographic slice images of plunge-frozen Magnetotactic bacteria between CTEM with energy filter and VPP without energy filter. Scale bars: $500 \mathrm{~nm}$. 